\title{
APPROXIMATION OF INDUCED AUTOMORPHISMS AND SPECIAL AUTOMORPHISMS
}

\author{
P. N. WHITMAN
}

\begin{abstract}
A class of measure-preserving invertible point transformations which admit approximations is defined. If $T$ is an automorphism which admits an approximation, conditions are given such that an induced automorphism and a special automorphism over $T$ again admit an approximation.
\end{abstract}

1. Preliminaries. Let $(X, F, \mu)$ be a measure-space isomorphic to the unit interval with Lebesgue measure. A measure-preserving invertible point transformation of $X$ is called an automorphism of $(X, F, \mu)$.

A finite ordered collection $\xi=\left\{C_{i}: 1 \leqslant i \leqslant q\right\}$ of pairwise-disjoint measurable sets in $X$ is called a partition. If the union of members of $\xi$ is $X$, then $\xi$ is called a partition of $X$. If $A \in F$, we write $A<\xi$ if $A$ is a union of members of $\xi$. If $\eta=\left\{B_{j}: 1 \leqslant j \leqslant p\right\}$ is a partition, we write $\eta \leqslant \xi$ if $B_{j} \leqslant \xi$ for $1 \leqslant j \leqslant p$. If $0<\beta<1$ we write $\xi \supset \beta \eta$ if each $B_{j} \in \eta$ contains a $C_{i} \in \xi$ with $\mu\left(C_{i}\right) \geqslant \beta \mu\left(B_{j}\right)$.

Let $\varepsilon_{X}$ denote the partition of $X$ into points. A sequence of partitions $\{\xi(n)\}$ converges to the unit partition, written $\xi(n) \rightarrow \varepsilon_{X}$, if for each $A \in F$, $\mu(A \triangle A(\xi(n))) \rightarrow 0$ as $n \rightarrow \infty$, where $A(\xi(n)) \leqslant \xi(n)$ and is such that $\mu(A \triangle A(\xi(n)))$ is a minimum.

The following definitions are due to Katok and Stepin [4] and Chacon [1], respectively.

Definition 1. An automorphism $T$ is said to admit a cyclic approximation by periodic transformations of the first kind (cyclic a. p. t. I) with speed $f(n)$, where $f(n)$ is a sequence of real numbers decreasing to zero, if there exists a sequence of partitions $\{\xi(n)\}, \xi(n)=\left\{C_{i}(n): 1 \leqslant i \leqslant q(n)\right\}$ such that:

1. $\xi(n) \rightarrow \varepsilon_{X} ;$ and

2. $\sum_{i=1}^{q(n)} \mu\left(T C_{i}(n) \triangle C_{i+1}(n)\right)<f(q(n))$ where $C_{q(n)+1}$ means $C_{1}(n)$.

Definition 2. An automorphism $T$ is said to admit a simple approximation if there exists a sequence of partitions $\{\xi(n)\}, \xi(n)=\left\{C_{i}(n): 1 \leqslant i \leqslant q(n)\right\}$ such that:

1. $\xi(n) \rightarrow \varepsilon_{X} ;$ and

2. $T C_{i}(n)=C_{i+1}(n), 1 \leqslant i \leqslant q(n)-1$.

It is known that automorphisms which admit a simple approximation are ergodic and have simple spectrum.

Received by the editors August 4, 1977.

AMS (MOS) subject classifications (1970). Primary 28A65. 
2. Induced automorphisms and special automorphisms. Let $T$ be an automorphism of $(X, F, \mu)$. Let $A \in F$.

Definition 3. The induced automorphism $T_{A}: A \rightarrow A$ is defined by

$$
T_{A} x=T^{k} x, \quad x \in A,
$$

where $k$ is the least positive integer such that $T^{k} x \in A$ (neglecting sets of measure zero).

Denote by $\mathbf{N}$ the set of nonnegative integers, and by $\mathbf{R}^{+}$the set of positive real numbers. Let $f: X \rightarrow \mathbf{N}$ be an integrable function. Put $B(k, n)=\{(x, n)$ : $x \in X, f(x)=k\}$. Put $X(f)=\cup_{k \geqslant 0} \cup_{n=0}^{k} B(k, n)$ and identify $X$ with the set $\cup_{k \geq 0} B(k, 0)$. We may regard each set $B(k, n), 0<n \leqslant k$, as a copy of $B(k, 0)$. Consequently, we may extend $\mu$ to $X(f)$ and form a normalised measure $\mu^{\prime}$ on $X(f)$ in the obvious way.

Definition 4. Let the transformation $T_{f}$ on $X(f)$ be defined by

$$
\begin{gathered}
T_{f}(x, n)=(x, n+1), \quad 0 \leqslant n<f(x), \\
T_{f}(x, f(x))=(T x, 0) .
\end{gathered}
$$

$T_{f}$ is called the special automorphism over $T$ built under the function $f$.

If $f$ is the characteristic function of a set $A \in F$, then the special automorphism $T_{f}$ is denoted $T^{A}$ and is called a primitive automorphism over $T$.

Order the sets $B(k, n), k \geqslant 0,0 \leqslant n \leqslant k$, lexicographically.

Definition 5. Let $\xi$ be a partition in $X$ such that every element of $\xi$ is contained in exactly one of the sets $B(k, 0)$ for some $k$. Then $\xi^{f}$ is the partition in $X(f)$ consisting of the elements $C \in \xi$, together with, for each $C \in \xi$, where $C \subset B(k, 0)$, a copy of $C$ in each of the sets $B(k, n), 0<n<$ $k$. The ordering on $\xi^{f}$ is that inherited from the sets $B(k, n), k \geqslant 0,0 \leqslant n \leqslant$ $k$.

It is easily seen that if $\xi(n) \rightarrow \varepsilon_{X}$, then $\xi^{f}(n) \rightarrow \varepsilon_{X(f)}$.

Recall that the measure algebra $(F, \mu)$ is a complete metric space with respect to the metric $d$ given by

$$
d(A, B)=\mu(A \triangle B), \quad A, B \in F .
$$

Goodson [3] has proved the following two theorems for induced and primitive automorphisms.

THEOREM 1. Let $T: X \rightarrow X$ be an automorphism which admits a simple approximation; then there is a collection of subsets of $X$, dense in $F$, such that the automorphisms $T^{A}$ and $T_{A}$ on any one of these sets also admit a simple approximation.

THEOREM 2. Let $T: X \rightarrow X$ admit a simple approximation with respect to a sequence of partitions $\{\xi(n)\}, \xi(n)$ having $q(n)$ elements, and suppose $A \in F$, with $\mu(A)>0$, can be approximated by sets $A(n) \subset A$ with $A(n) \leqslant \xi(n)$ and such that $q(n) \mu(A \backslash A(n)) \rightarrow 0$ as $n \rightarrow \infty$. Then $T^{A}$ and $T_{A}$ both admit a simple approximation. 
3. Main results. We show below that if $T$ admits a cyclic a.p.t.I with fast enough speed then both $T_{A}$ and $T^{A}$ admit a simple approximation, where $A$ is as in Theorem 2 . We require the following lemma.

LEMMA 1. Let $\{\zeta(n)\}$ be a sequence of partitions with $\zeta(n) \rightarrow \varepsilon_{X}$. Let $\{\xi(n)\}$ be $a$ sequence of partitions and let $\beta(n)$ be an increasing sequence of positive real numbers converging to 1 such that $\xi(n) \supset \beta(n) \zeta(n), n \geqslant 1$. Then $\xi(n) \rightarrow$ $\varepsilon_{X}$

Proof. Let $A \in F$ with $\mu(A)>0$. Since $\zeta(n) \rightarrow \varepsilon_{X}$, there is a sequence of sets $A(\zeta(n))<\zeta(n)$ such that $\mu(A \triangle A(\zeta(n))) \rightarrow 0$ as $n \rightarrow \infty$.

Let $D(n)=\bigcup\{C(n) \in \xi(n): C(n) \subset A(\zeta(n))\}$; then $\mu(A(\zeta(n)) \triangle D(n)) \leqslant(1-\beta(n)) \mu(\cup\{C(n): C(n) \in \xi(n)\})<1-\beta(n)$.

Consequently,

$$
\mu(A \triangle D(n)) \leqslant \mu(A \triangle A(\zeta(n)))+\mu(A(\zeta(n) \triangle D(n))) \rightarrow 0 \quad \text { as } n \rightarrow \infty .
$$

Hence,

$$
\mu(A \triangle A(\xi(n))) \leqslant \mu(A \triangle D(n))<0 \text { as } n \rightarrow \infty .
$$

The proof of the following result is based on techniques shown in [2].

THEOREM 3. Let $T: X \rightarrow X$ be an automorphism admitting a cyclic a.p.t. I with speed $f(n)=o\left(1 / n^{2}\right)$ with respect to a sequence of partitions $\xi(n) \rightarrow \varepsilon_{X}$, $\xi(n)=\left\{C_{i}(n): 1<i \leqslant q(n)\right\}$. Let $A \in F$ be approximated by sets $A(n)<$ $\xi(n)$ with $A(n) \subset A$ such that $q(n) \mu(A \backslash A(n)) \rightarrow 0$ as $n \rightarrow \infty$. Then $T_{A}$ and $T^{A}$ admit a simple approximation.

Proof. For each $i, 1 \leqslant i \leqslant q(n)$, we can write $C_{i}(n)=F_{i}(n) \cup G_{i}(n)$, where $T G_{i}(n) \subset C_{i+1}(n)$ and $T F_{i}(n) \cap C_{i+1}(n)=\varnothing, 1 \leqslant i \leqslant q(n) . C_{q(n)+1}(n)$ means $C_{1}(n)$.

Let $C_{1}^{\prime}(n)=C_{1}(n) \cap \cap_{i=1}^{q(n)} T^{-i+1} G_{i}(n)$ and put

$$
T^{i-1} C_{1}^{\prime}(n)=C_{i}^{\prime}(n), \quad 1 \leqslant i \leqslant q(n) .
$$

As in [2], it can be shown that

$$
\mu\left(C_{1}(n) \backslash C_{1}^{\prime}(n)\right)<\frac{1}{2} f(q(n)) .
$$

Thus,

$$
\mu\left(C_{1}^{\prime}(n)\right)>\mu\left(C_{1}(n)\right)-\frac{1}{2} f(q(n))=\mu\left(C_{1}(n)\right)(1-o(1 / q(n))) .
$$

Consequently, by Lemma $1, \xi^{\prime}(n) \rightarrow \varepsilon_{X}$, where $\xi^{\prime}(n)=\left\{C_{i}^{\prime}(n): 1 \leqslant i \leqslant q(n)\right\}$, and $T$ admits a simple approximation with respect to the sequence $\left\{\xi^{\prime}(n)\right\}$.

Let $A^{\prime}(n)=\cup\left\{C_{i}^{\prime}(n) \in \xi^{\prime}(n): C_{i}^{\prime}(n) \subset A(n)\right\}$. Then $A^{\prime}(n) \subset A$ and

$$
\begin{aligned}
\mu\left(A \backslash A^{\prime}(n)\right) & \leqslant \mu(A \backslash A(n))+\mu\left(A(n) \backslash A^{\prime}(n)\right) \\
& \leqslant \mu(A \backslash A(n))+\frac{1}{2} q(n) f(q(n)) \rightarrow 0 \text { as } n \rightarrow \infty .
\end{aligned}
$$

Furthermore,

$$
q(n) \mu\left(A \backslash A^{\prime}(n)\right) \rightarrow 0 \quad \text { as } n \rightarrow \infty
$$


Hence by Theorem 2, $T_{A}$ and $T^{A}$ admit a simple approximation.

Next, we give results for special automorphisms analogous to Theorems 1 and 2. From our results we are able to obtain a slightly weaker version of Theorem 2 for primitive automorphisms as a corollary.

THEOREM 4. Let $T: X \rightarrow X$ be an automorphism admitting a simple approximation with respect to a sequence of partitions $\xi(n) \rightarrow \varepsilon_{X}$. Let $f: X \rightarrow \mathbf{N}$ be integrable. Let $\xi(n)=\left\{C_{i}(n): 1 \leqslant i \leqslant q(n)\right\}$. Suppose that for each $C_{i}(n) \in$ $\xi(n), 1<i<q(n)$, there exists $k_{i}(n) \in \mathbf{N}$ such that

$$
\mu\left\{x \in C_{i}(n): f(x)=k_{i}(n)\right\} \geqslant(1-\delta(n)) \mu\left(C_{i}(n)\right)
$$

where $\delta(n)$ is a sequence of real numbers with $\delta(n)=o(1 / q(n))$. Then $T_{f}$ admits a simple approximation.

Proof. Let $B_{i}(n)=f^{-1}\left(k_{i}(n)\right)$. Put

$$
D_{1}(n)=\bigcap_{i=0}^{q(n)-1} T^{-i}\left(C_{i+1}(n) \cap B_{i+1}(n)\right) .
$$

Then

$$
\begin{aligned}
\mu\left(D_{1}(n)\right) & \geqslant \mu\left(C_{1}(n)\right)-q(n) \delta(n) \mu\left(C_{1}(n)\right) \\
& =\mu\left(C_{1}(n)\right)(1-q(n) \delta(n)) .
\end{aligned}
$$

Put $D_{i}(n)=T^{i-1} D_{1}(n), \quad 1 \leqslant i \leqslant q(n)$, and put $\eta(n)=\left\{D_{i}(n): 1 \leqslant i \leqslant\right.$ $q(n)\}$. Then $\eta(n) \supset(1-q(n) \delta(n)) \xi(n)$ by (1), and consequently $\eta(n) \rightarrow \varepsilon_{X}$ by Lemma 1 . Hence $\eta^{f}(n) \rightarrow \varepsilon_{X(f)}$ and $T_{f}$ admits a simple approximation with respect to $\left\{\eta^{f}(n)\right\}$.

In the case where $f$ is the characteristic function of a set $A$, Theorem 4 has the following weaker form of Goodson's result as a corollary.

COROllary 1. Let $T: X \rightarrow X$ be an automorphism which admits a simple approximation with respect to a sequence of partitions $\xi(n) \rightarrow \varepsilon_{X}, \xi(n)=\left\{C_{i}(n)\right.$ : $1 \leqslant i \leqslant q(n)\}$. Let $A$ be a measurable set approximated by sets $A(n)<\xi(n)$, $A(n) \subset A$, in the sense that $q(n)^{2} \mu(A \backslash A(n)) \rightarrow 0$ as $n \rightarrow \infty$. Then $T^{A}$ admits a simple approximation.

Proof. Let $f(x)=1, x \in A,=0, x \in X \backslash A$. Let $C(n) \in \xi(n)$. If $C(n) \subset$ $A(n)$, then clearly $\mu\{x \in C(n): f(x)=1\}=\mu(C(n))$. If $C(n) \subset X \backslash A(n)$, then

$$
\begin{aligned}
\mu\{x \in C(n): f(x)=0\} & =\mu(C(n))-\mu(C(n) \cap A) \\
& \geqslant \mu(C(n))-\mu(A \backslash A(n)) \\
& =\mu(C(n))-o\left(1 / q(n)^{2}\right) \\
& =\mu(C(n))(1-o(1 / q(n))) .
\end{aligned}
$$

Hence by Theorem $4, T_{f}=T^{A}$ admits a simple approximation.

We now give a density result for special automorphisms which is analogous to that of Theorem 1. 
THEOREM 5. The collection of functions $f: X \rightarrow \mathbf{N}$ which satisfy the conditions of Theorem 4 is dense in the set of integrable functions $g: X \rightarrow \mathbf{N}$ with the $L_{1}$ topology.

Proof. Suppose that $g: X \rightarrow \mathbf{N}$ is integrable, and takes values $k_{1}<k_{2}$ $<\ldots$ Put $G_{i}=g^{-1}\left(k_{i}\right), i \geqslant 1$. We assume, without loss of generality, that $\mu\left(G_{i}\right)>0, i \geqslant 1$. Now choose $m \in \mathbf{N}$ sufficiently large so that, given $\varepsilon>0$,

$$
\int_{X \backslash \cup \bigcup_{i=1}^{m} G_{i}} g d \mu<\frac{\varepsilon}{4} .
$$

Since $\xi(n) \rightarrow \varepsilon_{X}$, we can find $n$ such that there are $\xi(n)$-sets $E_{i}(n) \leqslant \xi(n)$, $1 \leqslant i \leqslant m$, which are pairwise disjoint and $\mu\left(E_{i}(n) \triangle G_{i}\right)<\varepsilon / 2 m k_{m}, 1 \leqslant i \leqslant$ $m$. Define $f(x)=k_{i}, x \in E_{i}(n),=0, x \in X \backslash \cup_{i=1}^{m} E_{i}(n)$. Then

$$
\begin{aligned}
\int_{X}|f-g| d \mu & =\int_{\bigcup_{i=1}^{m} G_{i}}|f-g| d \mu+\int_{X \backslash \bigcup_{i=1}^{m} G_{i}}|f-g| d \mu \\
& =\sum_{i=1}^{m}\left(\int_{G_{i}}|f-g| d \mu\right)+\int_{X \backslash \bigcup_{i=1}^{m} G_{i}}|f-g| d \mu \\
& <\sum_{i=1}^{m} \int_{G_{i} \cap E_{i}(n)}|f-g| d \mu+\int_{G_{i} \backslash E_{i}(n)}|f-g| d \mu+2 \int_{X \backslash \bigcup_{i=1}^{m} G_{i}} g d \mu,
\end{aligned}
$$

since $f(x)<g(x)$ if $x \in X \backslash \cup{ }_{i=1}^{m} G_{i}$. Thus by (2),

$$
\begin{aligned}
\int_{X}|f-g| d \mu & <\sum_{i=1}^{m} \int_{G_{i} \backslash E_{i}(n)}|f-g| d \mu+\frac{\varepsilon}{2}<\sum_{i=1}^{m} \int_{G_{i} \backslash E_{i}(n)}\left|f-k_{i}\right| d \mu+\frac{\varepsilon}{2} \\
& <\sum_{i=1}^{m} k_{m} \mu\left(G_{i} \triangle E_{i}(n)\right)+\frac{\varepsilon}{2}<\sum_{i=1}^{m} \frac{k_{m} \varepsilon}{2 m k_{m}}+\frac{\varepsilon}{2}=\varepsilon .
\end{aligned}
$$

The result below is analogous to Theorem 3 .

THEOREM 6. Let $T: X \rightarrow X$ be an automorphism which admits a cyclic a.p.t. I with respect to a sequence of partitions $\xi(n) \rightarrow \varepsilon_{X}, \xi(n)=\left\{C_{i}(n): 1 \leqslant i \leqslant\right.$ $q(n)\}$, with speed $g(n)=o\left(1 / n^{2}\right)$. Let $f: X \rightarrow \mathbf{N}$ be integrable, and suppose that for each $C_{i}(n) \in \xi(n), 1 \leqslant i \leqslant q(n)$, there exists $k_{i}(n) \in \mathbf{N}$ such that

$$
\mu\left\{x \in C_{i}(n): f(x)=k_{i}(n)\right\} \geqslant(1-\varepsilon(n)) \mu\left(C_{i}(n)\right),
$$

where $\varepsilon(n)$ is a sequence of real numbers with $\varepsilon(n)=o(1 / q(n))$. Then $T_{f}$ admits a simple approximation.

Proof. As in Theorem 3, we can show that there exists a sequence of partitions $\xi^{\prime}(n)=\left\{C_{i}^{\prime}(n): 1 \leqslant i \leqslant q(n)\right\}$ with $\xi^{\prime}(n) \rightarrow \varepsilon_{x}$ and $\mu\left(C_{i}(n) \backslash C_{i}^{\prime}(n)\right)$ $<\frac{1}{2} g(q(n))$ such that $T$ admits a simple approximation with respect to the sequence $\left\{\xi^{\prime}(n)\right\}$.

We show that $T$ and $\left\{\xi^{\prime}(n)\right\}$ satisfy the conditions of Theorem 4, from which it will follow that $T_{f}$ admits a simple approximation.

Let $B_{i}(n)=f^{-1}\left(k_{i}(n)\right)$. Then by (3), 


$$
\begin{aligned}
\mu\left(C_{i}^{\prime}(n) \cap B_{i}(n)\right) & =\mu\left(C_{i}(n) \cap B_{i}(n)\right)-\mu\left(\left(C_{i}(n) \backslash C_{i}^{\prime}(n)\right) \cap B_{i}(n)\right) \\
& \geqslant(1-\varepsilon(n)) \mu\left(C_{i}(n)\right)-\frac{1}{2} g(q(n)) \\
& \geqslant(1-\varepsilon(n)) \mu\left(C_{i}^{\prime}(n)\right)-\frac{1}{2} g(q(n)) .
\end{aligned}
$$

Put $\delta(n)=\varepsilon(n)+g(q(n)) / 2 \mu\left(C_{1}^{\prime}(n)\right)$; then $\delta(n)=o(1 / q(n))$. Hence, $\mu\left\{x \in C_{i}^{\prime}(n): f(x)=k_{i}(n)\right\}=\mu\left(C_{i}^{\prime}(n) \cap B_{i}(n)\right)>(1-\delta(n)) \mu\left(C_{i}^{\prime}(n)\right)$, and so by Theorem $4, T_{f}$ admits a simple approximation.

The above results give conditions for a special automorphism to admit a simple approximation. We now give conditions for a special automorphism to admit a cyclic a.p.t.I with a preassigned speed.

Definition 6. Let $T: X \rightarrow X$ be an automorphism. Then $T$ admits a cyclic a.p.t.I with speed $g(x)$ if $T$ admits a cyclic a.p.t.I with speed $g(n)$, where $g$ is a decreasing function from $\mathbf{R}^{+}$to $\mathbf{R}^{+}$with $g(x) \rightarrow 0$ as $x \rightarrow \infty$.

THeOREM 7. Let $T: X \rightarrow X$ be an automorphism which admits a cyclic a.p.t.I with respect to a sequence of partitions $\xi(n) \rightarrow \varepsilon_{X}, \xi(n)=\left\{C_{i}(n): 1 \leqslant i \leqslant\right.$ $q(n)\}$ with speed $g(x)=o\left(1 / x^{k}\right)$. Let $f: X \rightarrow \mathbf{N}$ be integrable with $f\left(C_{i}(n)\right)=$ $k_{i}(n) \in \mathbf{N}, 1 \leqslant i \leqslant q(n), n \geqslant 1$. Then $T_{f}$ admits a cyclic a.p.t.I with speed $o\left(1 / n^{k}\right)$.

Proof. Let $\xi^{f}(n)=\left\{C_{i j}(n): 1 \leqslant i \leqslant q(n), 0 \leqslant j \leqslant k_{i}(n)\right\}$, where $C_{i, 0}(n)=$ $C_{i}(n) \in \xi(n)$. For ease of notation put

$$
C_{i, k_{i}(n)+1}(n)=C_{i+1,0}(n) \text { and } C_{q(n)+1, k_{q(n)}+1}(n)=C_{1,0}(n) .
$$

Then

$$
\begin{aligned}
\sum_{i=1}^{q(n)} \sum_{j=0}^{k_{i}(n)} & \mu^{\prime}\left(T_{f} C_{i j}(n) \triangle C_{i, j+1}(n)\right) \\
& =\sum_{i=1}^{q(n)} \mu^{\prime}\left(T_{f} C_{i, k_{i}(n)}(n) \Delta C_{i+1,0}(n)\right) \\
& =\left(1+\int f d \mu\right)^{-1} \sum_{i=1}^{q(n)} \mu\left(T C_{i}(n) \triangle C_{i+1}(n)\right) \\
& <\left(1+\int f d \mu\right)^{-1} g(q(n)) .
\end{aligned}
$$

Let $p(n)$ be the number of elements in $\xi^{f}(n)$. Then

$$
\begin{aligned}
p(n) & =q(n)+\sum_{i=1}^{q(n)} k_{i}(n)=q(n)\left(1+\frac{1}{q(n)} \sum_{i=1}^{q(n)} k_{i}(n)\right) \\
& \leqslant q(n)\left(1+2 \mu\left(C_{1}(n)\right) \sum_{i=1}^{q(n)} k_{i}(n)\right),
\end{aligned}
$$

for $n$ sufficiently large, since $q(n) \mu\left(C_{1}(n)\right) \rightarrow 1$ and so $q(n) \mu\left(C_{1}(n)\right) \geqslant \frac{1}{2}$ for $n$ sufficiently large. 
We may assume without loss of generality that (4) holds for all $n$. Hence,

$$
p(n)<q(n)\left(1+2 \mu\left(C_{1}(n)\right) \sum_{i=1}^{q(n)} k_{i}(n)\right) \leqslant q(n)\left(1+2 \int f d \mu\right) .
$$

Now put $G(n)=g\left(n \cdot\left(1+2 \int f d \mu\right)^{-1}\right) .\left(1+\int f d \mu\right)^{-1}$. Then,

$$
\sum_{i=1}^{q(n)} \sum_{j=0}^{k_{i}(n)} \mu^{\prime}\left(T_{f} C_{i j}(n) \triangle C_{i, j+1}(n)\right)<\left(1+\int f d \mu\right)^{-1} g(q(n))<G(p(n))
$$

The conclusion of the theorem is now apparent.

By exactly the same reasoning we also have the following.

THEOREM 8. Let $T: X \rightarrow X$ be an automorphism which admits a cyclic a.p.t.I with respect to a sequence of partitions $\{\xi(n)\}, \xi(n)=\left\{C_{i}(n): 1<i<q(n)\right\}$ with speed $g(x)=\theta / x, \theta \in \mathbf{R}^{+}$. Let $f: X \rightarrow \mathbf{N}$ be integrable with $f\left(C_{i}(n)\right)=$ $k_{i}(n) \in \mathrm{N}, 1<i<q(n), n \geqslant 1$. Then $T_{f}$ admits a cyclic a.p.t.I with speed $G(n)=2 \theta / n$.

Proof. As for Theorem 7, noticing that

$$
\left(1+\int f d \mu\right)^{-1} g(q(n)) \leqslant \theta\left(1+2 \int f d \mu\right) / p(n)\left(1+\int f d \mu\right)<2 \theta / p(n) .
$$

It is known [1] that if $T$ admits a cyclic a.p.t.I with speed $\theta / n$, where $\theta<1$, then $T$ has simple spectrum.

We do not know if, in general, a sufficient condition for $T_{f}$ to have simple spectrum is that $T$ has simple spectrum.

\section{BIBLIOGRAPHY}

1. R. V. Chacon, Approximations and spectral multiplicity, Lecture Notes in Math., vol. 160, Springer-Verlag, Berlin and New York, 1970, pp. 18-27.

2. R. V. Chacon and T. Schwartzbauer, Commuting point transformations, Z. Wahrscheinlichkeitstheorie und verw. Gebiete. 11 (1969), 277-287. MR 39 \#2939.

3. G. R. Goodson, Induced automorphisms and simple approximations, Proc. Amer. Math. Soc. 54 (1976), 141-145. MR 52 \# 10997.

4. A. B. Katok and A. M. Stepin, Approximations in ergodic theory, Russian Math. Surveys (5) 22 (1967), 77-102. MR 36 \#2776.

Department of Mathematics, University of Sussex, Falmer, Brighton, England

Current address: University of Witwatersrand, Jan Smuts Avenue, Johannesburg, South Africa 